\author{
H. Viet Tran \\ Etienne H. Bovey \\ Sylvie Uffer \\ Leonidas Zografos
}

\section{Peripapillary choroidal neovascularization associated with melanocytoma of the optic disc: a clinicopathologic case report}

Received: 1 November 2005

Revised: 29 December 2005

Accepted: 9 January 2006

Published online: 8 March 2006

C) Springer-Verlag 2006
H. V. Tran · E. H. Bovey $(\bowtie)$.

S. Uffer · L. Zografos

Jules Gonin University Eye Hospital, 15, Av. de France,

1004 Lausanne, VD, Switzerland

e-mail: Etienne.bovey@ophtal.vd.ch

\begin{abstract}
Background: Melanocytoma of the optic disc is a benign melanocytic tumor that rarely causes visual impairment. We report a case of a melanocytoma of the optic disc with a decreased vision related to a peripapillary choroidal neovascular membrane (PCNVM) that was successfully treated by submacular surgery. Methods: A 45-year-old southern European patient had a melanocytoma of the optic disc in his left eye with vision of 20/100. Fluorescein angiography demonstrated a PCNVM impeding the fovea associated with a subretinal hemorrhage. Results: The patient underwent a
\end{abstract}

complete vitrectomy and removal of the PCNVM. Subsequently, the subretinal hemorrhage disappeared and visual acuity improved to $20 / 25$. Visual acuity remained good for a period of 14 months' follow-up without any recurrence of neovascular membrane. Conclusions: Submacular surgery is a potentially effective treatment for large PCNVM associated with a melanocytoma of the optic disc.

Keywords Melanocytoma $\cdot$ Optic nerve $\cdot$ Choroidal neovascular membrane Submacular surgery . Histopathology

\section{Introduction}

Melanocytoma is a variant of melanocytic nevus and is most often localized in the optic disc, although it can occur anywhere in the uveal tract $[5,7]$. Melanocytoma of the optic disc is a benign melanocytic tumor that rarely causes visual impairment, but it can cause an afferent pupillary defect and a visual field abnormality [2, 4]. When visual loss occurs, it is more likely due to necrosis of the benign tumor and loss of optic nerve function or compression of adjacent blood vessels producing retinal vein or artery occlusion rather than to malignant transformation [8]. Proliferation of choroidal neovascularization on the surface of these lesions has been described in a limited number of cases and formerly constituted an indication for enucleation due to suspected melanoma $[1,6]$. To our knowledge, this is the first case of peripapillary choroidal neovascular membrane (PCNVM) associated with a melanocytoma of the optic disc that has successfully been treated by submacular surgery.

\section{Case report}

A 45-year-old southern European patient was referred to our department for evaluation and treatment of a recently discovered pigmented mass on the optic disc of the left eye that had been causing a severe loss of visual function for 1 month. Best corrected visual acuity of the affected eye was 20/60. Slit lamp examination was normal and intraocular pressure was $16 \mathrm{mmHg}$ in both eyes. Visual field showed a peripapillary scotoma and a small superior temporal defect. The lesion was located in the temporal sector of the optic nerve and had a choroidal extension, and an intraretinal component with a feathery margin, compatible with its location in the nerve fiber layer (Fig. 1a). A large subretinal neovascular membrane was situated between the optic nerve and the fovea. On both fluorescein and indocyanin green (ICG) angiography the neovascular membrane extended from the optic nerve to half the surface of the fovea. The tumor's thickness was $2.0 \mathrm{~mm}$ on B-scan ultrasonography. The diagnosis of a presumed melanocy- 
toma complicated by neovascularization phenomena was retained and since there was a relatively good residual visual acuity, a periodic observation was decided upon. Three months after the initial examination, visual acuity, the size, and the shape of both tumor and PCNVM remained unchanged.

After 5 months of follow up, visual acuity decreased to 20/100 and an increased size of the arcuate scotoma and peripapillary defect was observed. Fundus examination showed a large subretinal hemorrhage, and fluorescein and ICG angiography revealed an enlarged extension of the PCNVM to the entire fovea (Fig. 1b). There was no posterior vitreous detachment. The tumor dimensions remained stable. The extensive size of the PCNVM was not accessible to laser treatment as the surface to be treated would have impinged on the macula. Thus, we decided to perform surgical excision of the PCNVM.

A three-part pars plana vitrectomy was performed by one of the authors (EHB). The retinotomy was made at the superior aspect of the neovascular complex, parallel to the nerve fiber bundle, and the neovascular membrane was grasped and removed in a single piece. The posterior hyaloid was adherent to the subretinal hemorrhage and to the surrounding retina. The histopathologic examination showed a single ingrowth stalk of a dense, vascularized fibrous tissue, situated in the interface of a degenerated retinal pigment epithelium and an atrophic, disorganized gliotic retina (GFAP positive). Neovessels did not invade the retina and Bruch's membrane was not included in the neovascular complex (Fig. 2a,b). A large vascularized cluster of densely pigmented polyhedral melanocytic cells was present beneath and above the retinal pigment epithelium. The nuclei were small and regular with no prominent nucleoli. No mitotic figures were seen (Fig. 2c). On immunohistochemistry, the cells stained positive for HMB45 and Melan-A (Fig. 2d).

Fourteen months after surgery, visual acuity improved to 20/25 and the visual field defect decreased (Fig. 1c). The retina was attached, the dimensions of the tumor remained stable and fluorescein angiography did not reveal any recurrence of the PCNVM.

\section{Discussion}

Development of choroidal neovascularization related to melanocytoma is a rare phenomenon and it can lead to severe loss of visual function. This situation can be differentiated from a malignant transformation by the constant size of the tumor. We are unaware of previous reports of PCNVM related to a melanocytoma of the optic disc that was successfully removed by submacular surgery.

Peripapillary melanoma with disruption of the Bruch's membrane can mimic a melanocytoma of the optic disc. It has one choroidal part and an extension into the vitreous cavity at the junction of the retinal pigment epithelium and the optic disc. However, peripapillary melanoma with disruption of the Bruch's membrane would have an extended choroidal part associated with alterations of the pigment epithelium, lipofuscin deposit, and show as a
Fig. 1 a At presentation. Fundus photograph: serous retinal detachment surrounded by lipid deposits overlying the choroidal component of the tumor. Fluorescein angiography, arterio-venous phase: extensive neovascular membrane on the surface of the choroidal localization of the tumor. Indocyanin green (ICG), late phase: diffusion of the dye beyond the limits of the lesion. b Five months later, before surgery. Fundus photograph: large subretinal hemorrhage. Fluorescein angiography, arteriovenous phase: progression of the neovascular membrane invading the fovea. Venous phase: leakage of the dye. c Fourteen months after surgery. Fundus photograph: the dimensions of the tumor remained stable. Fluorescein angiography, early and late phase: occlusion of the choroidal neovascularization and absence of diffusion of the dye. Alterations of the retinal pigment epithelium. Reattachment of the retina

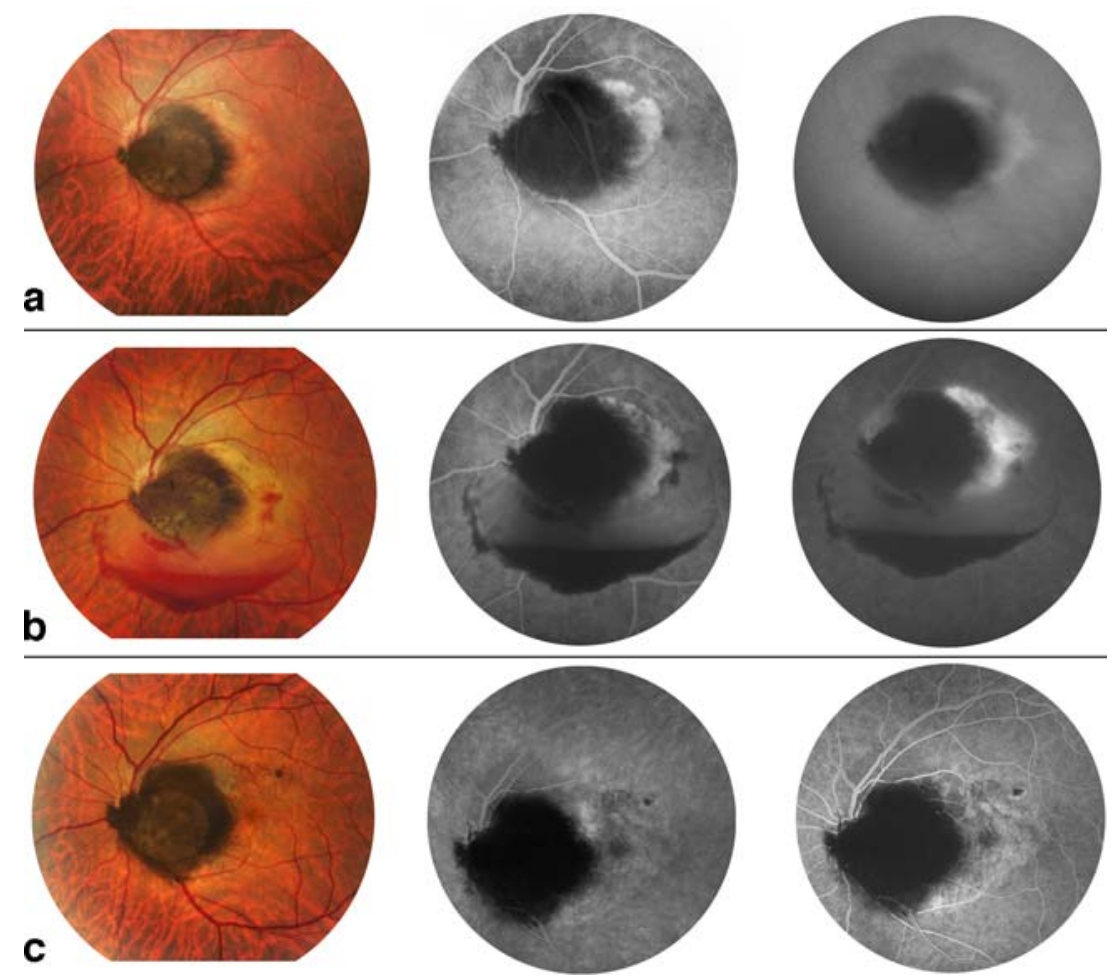


Fig. 2 Histopathology. a Part (A) of the membrane was removed with the overlying retina; part $(B)$ without retina has folded above part $A$ during the histologic preparation. Infolded neovascular membrane with a cluster of pigmented melanocytic cells (asterisk) situated beneath and above the retinal pigment epithelium (arrow; HE; original magnification, $\times 4$ ). b Fibrovascular membrane $(C N V)$ situated between a degenerated retinal pigment epithelium (arrow) and a gliotic retina (asterisk). (HE; original magnification, $\times 40)$. c Bleached preparation showing polyhedral tumor cells with bland nuclei. (HE; original magnification, $\times 100$ ). d Positive staining of the tumor cells for HMB45 (original magnification, $\times 100$ )

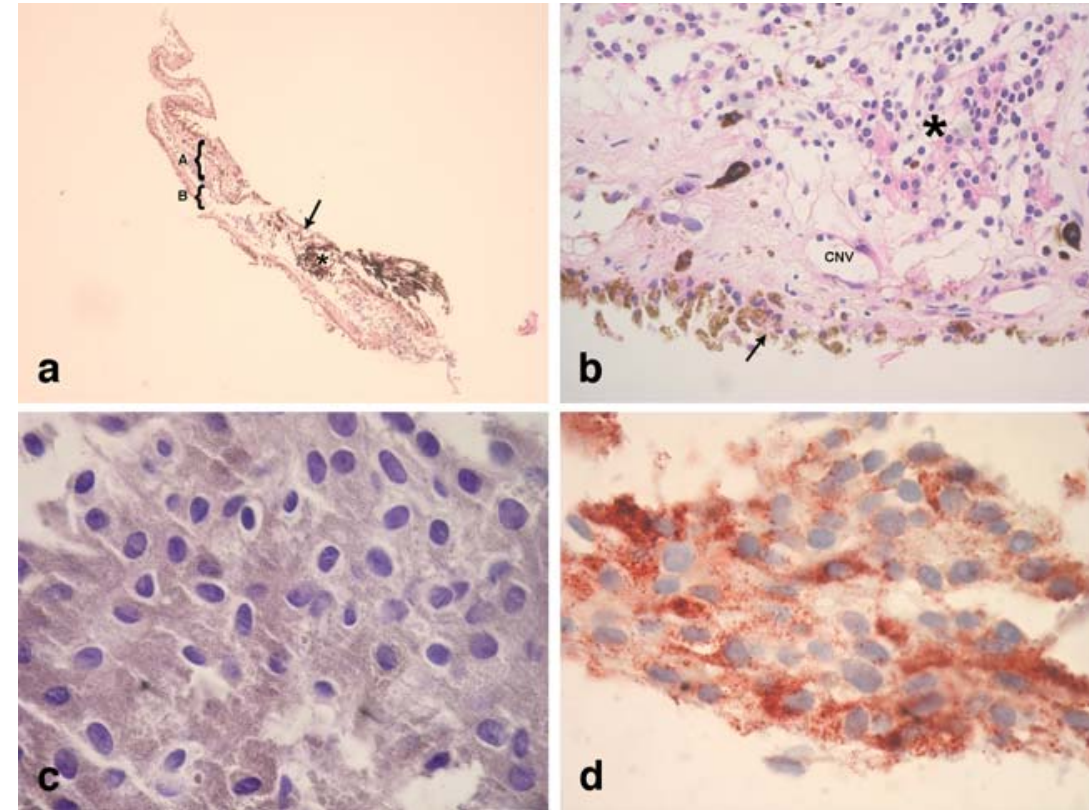

pinpoint on fluorescein angiography. The vitreous part would be darker, more abruptly elevated, and more likely to have associated exudative retinal detachment. Finally, because of the fast growth of the tumor, a retinal nerve fiber layer infiltration of pigment is absent.

The histopathology of uveal melanocytoma was clearly defined by Juarez and Tso in 1980 [3]. Two types of cells were recognized. Type 1 , which generally predominates, is a larger polyhedral, densely pigmented cell, with a small, bland nucleus, occasionally showing a small nucleolus. Type 2 is a smaller, less pigmented spindle cell, with a more conspicuous nucleolus. Neither type shows mitoses. These two types of cells are generally intermingled. In our case, the bleached preparation of the tumor showed the typically abundant cytoplasm and the small, bland nucleus of the type 1 cell.
Because PCNVM usually begins well away from the fovea, patients suffering from these neovascular membranes typically remain asymptomatic until the extension of the lesions themselves or associated subretinal fluids or blood begin to impinge on the fovea. In patients with good residual visual acuity, the laser required for the ablation of these large neovascular complexes close to the optic nerve and the fovea would destroy the maculo-papillar bundle, and endanger the optic nerve. Therefore, in selected cases submacular surgery is a valuable therapeutic modality.

Acknowledgement We would like to thank Fromhold Samuel for his technical support. This work was not sponsored by any commercial organization. Financial interest: none. Grant: none.

\section{References}

1. Howard GM, Forrest AW (1966) Melanocytoma of the disc complicated by hematoma under retinal pigment epithelium. Trans Am Acad Ophthalmol Otolaryngol 70:281-285

2. Joffe L, Shields JA, Osher RH, Gass JD (1979) Clinical and follow-up studies of melanocytomas of the optic disc. Ophthalmology 86:1067-1083

3. Juarez CP, Tso MO (1980) An ultrastructural study of melanocytomas (magnocellular nevi) of the optic disk and uvea. Am J Ophthalmol 90:48-62
4. Osher RH, Shields JA, Layman PR (1979) Pupillary and visual field evaluation in patients with melanocytoma of the optic disc. Arch Ophthalmol 97:1096-1099

5. Reidy JJ, Apple DJ, Steinmetz RL, Craythorn JM, Loftfield K, Gieser SC, Brady SE (1985) Melanocytoma: nomenclature, pathogenesis, natural history and treatment. Surv Ophthalmol 29:319-327

6. Rubin ML (1976) Disciform lesion overlying melanocytoma simulating progression of choroidal melanoma. Trans Am Ophthalmol Soc 74:282-294
7. Zimmerman LE (1965) Melanocytes, melanocytic nevi, and melanocytomas. Invest Ophthalmol 34:11-41

8. Zografos L (2002) Melanocytomes. In: Tumeurs intraoculaires. Rapport SFO (ed) Masson, Paris, pp 82-94 